\title{
An approach to digital literacy for adults: the EuNIC proposal
}

\author{
Manuela Delfino, Maria Ferraris, Donatella Persico and Francesca Pozzi \\ CNR, Institute for Educational Technology - Via de Marini, 6 - 16149 \\ Genova, Italy \{delfino, ferraris, persico, pozzi\}@itd.cnr.it, \\ WWW home page: http://www.itd.cnr.it/
}

\begin{abstract}
Recent surveys point to the need to enhance digital literacy competences among adults. Still, digital literacy courses addressing adults usually face a number of problems due to the lack of common backgrounds and purposes among learners. This paper illustrates NIC (Nucleo Informatico Concettuale - Conceptual Informatics Nucleus), an approach to adult digital literacy that focuses on few key concepts and skills and adopts a problembased approach. NIC, which was developed and widely tested in Italy, has been recently transferred to a European context by the EuNIC project. The main results of this process are reported together with some reflections about strong points and weaknesses of the suggested approach and, more in general, about critical aspects of adult digital literacy actions.
\end{abstract}

\section{Introduction}

Although digital literacy is considered one of the key competences that all individuals need for fully participating in the information society [1], a large part of the European population still seems to lack even the most basic skills which underpin that competence. The 2005 Eurostat survey, which focused on what they call "eskills" or "computer skills" of Europeans, was carried out on a sample of 200.000 people aged 17 to 74 and showed that $37 \%$ of the sample had no computer skills at all, $15 \%$ had a low level of competence, $26 \%$ a medium level and $22 \%$ declared to be familiar with a wide range of computer uses [2]. These values vary remarkably according to the country of origin, level of education and age. In young people the lack of e-skills drops to $10 \%$ and to $4 \%$ in students; in the latter group all countries report the highest level of e-skills (i.e., an average of $43 \%$ versus $22 \%$ in the whole sample). This survey only supplies information on basic e-skills, whose mastery is only a prerequisite of digital literacy. Nonetheless, the Eurostat data clearly show that social groups of people outside education and training circuits and in the 
working age, are more likely to be "excluded" by the "digital world" $-46 \%$ of people aged between 24 and 55 has either a very low level of e-skills or none.

Hence there's a need to close this gap that can be addressed by increasing ICT access opportunities and by promoting initiatives aimed at the development of digital literacy in this age range.

Unlike school education, adult training often takes place over a short period of time, outside a real context, and involves people with very different backgrounds and motivations. With these constraints, an introductory course is unlikely to change a neophyte into an experienced and fluent ICT user. Being "fluent" requires substantial training, experience based on solving authentic problems and a number of nontechnological prerequisites [3]. Moreover, as technology tends to change in a very fast way, what we mean by "competency in a technological environment" constantly evolves [4: 55]. In other words, digital literacy cannot be intended as a well defined set of competences acquired once and for all, indeed, it entails a continuous learning process. As a consequence, a major aim of the initial training should be to put the beginners in the line to become digital literates, by fostering their capability to learn something new in an autonomous way. To this purpose, the approach adopted in the various courses based on the ECDL syllabus [5] does not seem to be the most suitable since it provides a step by step and largely rote acquisition of different skills in the use of computers and their applications. Typically, after courses based on howto-do approaches, it is quite common to notice that participants find it hard to face unknown situations and cannot transfer ways of working from a familiar situation to an unknown one. A plausible hypothesis is that, no matter how continuous the evolution of digital literacy is, there is a conceptual and operative basic core to be mastered which is crucial to acquire new knowledge. If this is true, too much emphasis on computer technicalities may even prevent the acknowledgement of this basic core of concepts and skills.

Moreover, this approach, as Eisenberg and Johnson write, "addresses the "how" of computer use, but rarely the "when" or "why." Participants may learn isolated skills and tool uses, yet many will lack an understanding of how those various skills fit together to solve problems and complete tasks" [6]. This suggests that, in a digital literacy action, problems and tasks should drive the learning process instead of being used just as examples of how to operate with the computer.

All these considerations are at the basis of NIC, an approach to adult digital literacy which was recently transferred to other European contexts within an EC project. In the following, the description of the approach, the transfer strategy at European level, and the outcomes of pilot courses in various countries are discussed.

\section{NIC: an approach to adult digital literacy}

NIC, the acronym of "Nucleo Informatico Concettuale" (Conceptual Informatics Nucleus), is a face to face course for adults designed in 2001 by the Institute for Educational Technology of the Italian National Research Council (ITD) and used in the local training system of the Provincia of Genova (District of Genova).

The aim of NIC is to train a "wise beginner user", i.e. a user who is able to interact with the computer to carry out simple tasks (write a text, send an e-mail, 
search and browse web pages, etc.); who understands how technology works and has an idea of its limits and potential. Most importantly, wise beginners can improve their computer competence in an autonomous way (e.g., by find out and learn how to use new functions of an application).

The NIC approach is not "technology oriented", but rather "concept and problem oriented". Instead of teaching how to use specific environments and developing skills that may turn out to be fragile, superficial and technology-dependent, this approach is based on training to master a set of conceptual and operative tools underlying all digital environments and use them to solve meaningful problems.

For this reason, an important feature of the NIC approach is its focus on a small nucleus of basic concepts and skills, which are considered essential to interact with the computer and to underpin new, autonomous learning. This nucleus was distilled during the design phase of the course by refining the definition of wise beginner user, by analyzing naives' difficulties and by involving experts in the multiple revision of a draft inventory of conceptual, terminological and operative knowledge.

A second essential feature of the NIC approach is the use of a problem-based method in order to make students transparently learn "why", "when" and "how" to use the main computer functions and applications to solve real or realistic problems. Consistently with this approach, a large portion of the NIC course is devoted to the development of a project that requires participants to face a number of different tasks in a complex situation usually chosen by the teacher according to the competences, interests and professional background of the group of learners involved.

Another key point in the NIC methodology lies in the attention devoted to the promotion of habits and attitudes that play a key role in beginner's autonomy (e.g., using "trial and error" strategies, reading and understanding computer messages, using the online help, working out procedures rather than trying to memorize them). These behaviours cannot certainly be taught, but can be encouraged and solicited through activities and teaching strategies aimed at stimulating or inducing them.

\subsection{NIC contents and structure}

According to the above features, the NIC course is organized into three stages:

1) the Central core that, in a short period of time, aims to the acquisition of fundamental skills (e.g., how to use mouse and keyboard, access files and folders, manipulate windows) and the introduction of core concepts, mostly essentials about the way computers work and the interplay of their main hardware and software components. This is a crucial stage, as it concerns concepts and skills that are prerequisite for further learning.

2) the Immersion, that entails the development of a project requiring the integrated use of different applications (word-processor, web-browser, e-mail, database, spreadsheet, multimedia presentation). The aim is not to provide detailed and exhaustive competences on the use of these programs, but to consolidate the core concepts introduced in the first phase, to demonstrate recurrent patterns common to different applications and to develop awareness of the types of problems that can be solved with each application. The project that drives this stage is chosen by the NIC teacher, according to the learners' interests and backgrounds, provided that all the above mentioned applications are used. During the first edition of the NIC course, in 
2001, the project concerned the introduction of the new currency, a topical issue at that time. This is how the problem was posed: "In a few months we will change our currency, from Lira to Euro. You all work in a bank. Your director asks you to conduct a survey to find out clients' opinions and knowledge about Euro. You should prepare a questionnaire, distribute it, collect the results, analyze them and present a final report". This problem required learners to use a word processor to draw up a questionnaire; to use e-mail to distribute it; to use Internet in order to retrieve information (texts, images, etc.); to design and populate a data-base with the answers to the questionnaires; to transfer data from a data-base to a spreadsheet for analysing them and creating charts; to prepare a presentation and illustrate the survey results.

3 ) the Emersion stage, aiming to support informed choices of future training initiatives and to widen the course horizons, by setting computing in an historical framework and giving practical suggestions to foster an autonomous computer use.

\subsection{NIC teaching strategy and instructional materials}

The way in which contents and activities of the course are presented to students is crucial for the success of the initiative. The NIC approach includes some specific teaching strategies: (1) a very gradual approach to new concepts and operative skills to foster the development of stable learning and avoid cognitive overload; (2) frequent switches from explanations to practice, to reify new concepts through hands-on activities or use practical tasks to introduce new concepts; (3) strong emphasis on stable concepts and skills rather than on technology-dependent aspects; this can be obtained by avoiding unneeded technical terms, discouraging rote learning, stressing the importance of giving priority to the problem and its definition before focusing on the technical ways to solve it; (4) students' engagement in activities that promote motivation and good habits.

To support the learning process, the NIC course provides three kinds of materials: (a) a set of interactive tutorials aiming to help the development of basic skills (e.g., the use of the mouse) or to support the comprehension of some key concepts (e.g., files organisation in folders); (b) the student worksheets, a collection of printed learning materials provided to students at the end of each lesson; (c) a set of files to be used by students for carrying out exercises and activities.

After a first tuning in 2001, the course was run in Liguria (Italy) in more than 160 editions, funded by the European Social Fund or by the Provincia di Genova, and involved more than 1700 participants. The good results obtained and the exportability demonstrated by the approach encouraged its further dissemination.

\section{Transferring NIC at European level: the EuNIC experience}

The EuNIC project, run from January 2006 to June 2007, aimed at turning the NIC principles and practice into a European approach to digital literacy for adults [7] thanks to the cooperation of five countries (Italy, Portugal, Greece, Latvia, Bulgaria).

The transfer occurred by collaboratively adapting and tuning the approach to the partners' needs, and subsequently testing it in each partner country through pilot 
courses. These courses were meant to be managed by pioneer teachers who would become the experts in their country for the EuNIC approach. All partners addressed the EuNIC pilot courses to adults, aged between 21 and 65, both unemployed and employed, with different professional backgrounds (e.g., housewives, farmers, teachers). This was compatible with the NIC approach, which was originally designed for adults with little or no computer skills and was independent of their profession and experience. The EuNIC transfer experiments involved 13 teachers and 60 participants across the four target countries. In the following, the actions undertaken by the consortium are described and the project results are discussed.

\subsection{Adapting materials and training teachers}

In order to transfer the NIC approach, the original materials underwent a revision which included an evaluation of the core concepts to verify their validity after five years from their formulation and the updating of technology-dependent aspects.

In particular, some Italian teachers experienced with the NIC approach carried out an analysis of the materials, with the aim to identify weaknesses. Furthermore, the original materials - based on Windows ME - were updated according to new software versions and to new devices available (Windows XP was chosen). Finally, aspects peculiar to the Italian culture were modified and adapted to a European audience: after their translation into English, all materials were translated into the national language and customized to local needs by each partner.

The teachers, selected on the basis of the local contexts and affordances, played a key role in the process of methodological transfer because they were in charge of running the EuNIC courses, tuning the approach according to the context and collecting the data needed to evaluate the experiment [8]. In case of success, dissemination of the approach at national level would have to rely on these teachers for the training of more EuNIC teachers. To help them acquire a deep understanding of the methodology and the learning materials, particular attention was devoted to the development of a Teacher's guide containing guidelines on how to organize each lesson, how to handle each kind of activity, what material to use, and so on.

The approach was presented to the pioneer teachers in an online course, during which they were required to perform individual study and collaborative activities.

\subsection{The EuNIC evaluation model}

The EuNIC evaluation model was conceived as an integral part of the approach itself and was aimed to provide the EuNIC partners with methods and tools to monitor and evaluate the pilot courses. It intended to gather data about the participants to pilot courses, to assess course quality as perceived by students and teachers, and to evaluate the course effectiveness in terms of learning achievements. The evaluation model focuses on three elements: the context, the teachers and the students.

The first group of indicators, the Contextual indicators, is aimed at investigating the level of flexibility of the approach. In other words, these indicators are intended to answer the question whether and to what extent it is possible to apply the same course structure and schedule in other countries. They were identified throughout the 
project development and during a conclusive workshop, by discussing the critical aspects of the project and how they could be faced with project partners and teachers. The second group, the Teacher centred indicators, aims to understand what EuNIC teachers thought about the materials and the friendliness and transferability of the approach. The third group, the Student centred indicators, is aimed at gathering data about the students' impressions on the course and the level of learning achievements. The second and third type of indicators were estimated through a set of tools aimed at gathering qualitative and quantitative information from all pilot courses in a systematic and consistent way. These tools included pre-course and end-of-course questionnaires for both teachers and students, a teacher logbook, a final student assessment test and teacher guidelines on how to deliver and mark the final test. In accordance with the approach, the final assessment test consisted in practical tasks entailing the acquisition of the basic concepts and skills.

While acknowledging the exploratory nature of the EuNIC project, the following section reports the major outcomes obtained by the application of the evaluation model to the pilot courses [9]. They are the basis for a reflection on the effectiveness of the transfer process and provide suggestions for further improvement and dissemination of the approach.

\section{Project results}

\subsection{Learning outcomes}

The learning outcomes of the pilot courses turned out to be very satisfactory. In a range from 1 to 3 , students from all the partners countries obtained a mean test rate of $2.86(\mathrm{SD}=0.34)$. Even in those tasks that required students a high degree of autonomy (e.g., find out a way to carry out a given task), the mean test rate was definitely high $(\mathrm{M}=2.8, \mathrm{SD}=0.4)$. No major variations seemed to occur when studying the distribution according to the country, the age, or the educational qualification. Quite interestingly, students who had initially declared to be totally unskilled with computers, obtained very good results too $(\mathrm{M}=2.88, \mathrm{SD}=0.38)$. One more favourable element emerging from the final assessment results is that students' evaluation of their confidence in carrying out each task is positively correlated to test results for those tasks (2-tailed Pearson correlation .63; $\mathrm{P}<0.01$ ). This suggests that students were aware of their ability, which is an important component of learning autonomy, a major aim of the course.

Students' perceptions of their achievements was also quite high, as testified by the answers to the following questions of the final questionnaire: "To what extent do you feel you are now an autonomous computer user?" $(\mathrm{M}=3.83, \mathrm{SD}=0.84$, range from 0 to 5$)$ and "Have you learnt something useful?" $(\mathrm{M}=4.43 ; \mathrm{SD}=0.80)$. 


\subsection{Course acceptance and materials transferability}

Since the very beginning of the project, there was also a very positive acceptance of the NIC approach on the side of the project partners. The partner delegates who participated in this first meeting showed great appreciation for the approach and they all underlined the fact that, in their countries, the need for such a non-technical approach was very strongly felt and that their institution had joined the project in order to break with a past of unsuccessful, technology-oriented digital literacy courses. Consistently with this initial attitude of the partners, both students' and teachers' perception about the course, as it emerged from the end-of-course questionnaires, show a positive overall evaluation of the learning materials.

In particular, in a range from 0 to 5 , students assigned a mean of 4.31 to overall course quality, 3.98 to learning material quality and 3.92 to balance/coherence of contents. No particular variations were registered according to the students countries.

In a range from 0 to 3 , teachers mean ratings of the effectiveness of the EuNIC approach was 2.67 for the Central core, 3 for the Immersion and 2.83 for the Emersion stage ( $\mathrm{SD}=0.40 ; 0.0 ; 0.41$ respectively). Teachers' opinions on the EuNIC materials were also very comforting: the mean ratings of the students' worksheets and of the teacher guide were $2.91(\mathrm{SD}=0.30)$, while the mean rating of the tutorials was $2.92(\mathrm{SD}=0.33)$. Other data from the teachers final questionnaires support a good degree of transferability of the materials to different contexts. First of all, teachers rated quite low the difficulties in understanding the NIC approach $(\mathrm{M}=0.5$, $\mathrm{SD}=0.84$ for the Central core phase; $\mathrm{M}=1.17, \mathrm{SD}=0.75$ for the Immersion phase; $\mathrm{M}=1.0, \mathrm{SD}=0.63$ for the Emersion). Furthermore, their understanding of the approach was confirmed during the teacher training course, when they were asked to devise a problem relevant for their target population. In Bulgaria the target population was composed of teachers and so the proposal concerned the evaluation of teachers' achievements. In Greece the target population consisted of housewives and farmers: they proposed to carry out a survey on the ecological habits of Greek families and an investigation on how farmers may access European funding. Overall the EuNIC teachers showed the ability to choose topics motivating students and well suited to the course purposes, thus demonstrating their understanding of the key features of the method and of the approach flexibility.

\section{Conclusions}

The EuNIC project intended to probe the transferability of the NIC approach through a number of pilot courses in order to come out with some indications laying the foundations for further work in the area. Even though the exploratory nature of the EuNIC project doesn't allow to say a final word about the transferability of the NIC approach, the process of adaptation, updating and localization of materials, and the training of teachers, suggested some considerations on the approach validity.

Transferring the approach required a revision on two axes: the axe of space, in order to extend the use of the NIC approach from a local context to different European countries, and the axe of time, in order to update the materials according to technological developments occurred in the five years after its first design. 
During the revision, while the materials related to the applications needed to be updated on both the axes, none of the experts - researchers and teachers - felt the need to alter the problem-based approach, the core of key skills and concepts or the teaching strategy. In other words, the essence of the NIC approach remained unaltered. Probably, due to technological developments, future revisions will need to have greater impact, especially on the choice of applications to be introduced in the course. For example, while so far the MS Office suite has been the classical set of applications taught in courses for beginners, it is likely that other types of tools (e.g., multimedia and communication applications) will deserve more attention in future.

A final consideration concerns the fact that digital literacy involves nontechnological key competences too. For instance, in order to effectively retrieve information from the web, just knowing how to use a search engine is not enough, since other abilities are required (e.g., to be able to select adequate key words, to discriminate between low and high quality information). Indeed, courses addressing adults with different backgrounds often face problems when participants lack some of the necessary non digital knowledge (e.g., linguistic, mathematical, logical thinking). On one hand, this strengthens the idea that courses for beginners should focus not only on few concepts and problems selected according to the participants' previous competences, but also on the above-mentioned non technological skills. More generally, this points to the need that in the field of adult lifelong learning, technology introductory courses should be integrated with actions concerning other kinds of literacies, recognizing the idea that there is only one literacy where digital and non digital competences are intertwined.

\section{References}

[1] European Parliament and Council, Recommendation of the European Parliament and of the Council, of 18 December 2006, on key competences for lifelong learning Official Journal of the European Union L.394/5, 2006.

[2] Eurostat, How skilled are Europeans in using computers and the Internet?. Collection Statistic in focus, 17, 2006.

[3] K. Williams, Literacy and computer literacy: Analyzing the NRC's being fluent with information technology. The Journal of Literacy and Technology, 3(1) (2003).

[4] U. Bunz, Growing from computer literacy towards computer mediated communication competence: evolution of a field and evaluation of new measurement instrument. Information Technology, Education and Society, 4(2), 53-84 (2003).

[5] European Computer Driving Licence, (Apr. 6, 2008); http://www.ecdl.com/.

[6] M.B. Eisenberg, and D. Johnson. Learning and Teaching Information

Technology - Computer Skills in Context, ERIC Digest, ED465377 (2002; updated version in 2006).

[7] CNR-ITD, Report 2:EuNIC:towards the definition of a European approach to digital literacy.T.R.01/07 [http://www.eu-nic-project.eu/pdf/EuNIC\%202Report.pdf] [8] CNR-ITD, Report 3:Supporting the EuNIC teachers. T.R.02/07

[http://www.eu-nic-project.eu/pdf/EuNIC_Report3.pdf]

[9] CNR-ITD, Report 4:EuNIC transferability strategy. T.R. 03/07 [http://www.eunic-project.eu/pdf/EUNIC\%204Report.pdf] 